\title{
molecules
}

ISSN 1420-3049

http://www.mdpi.org

Full Paper

\section{Microwave-promoted Facile and Efficient Preparation of $N$-(alkoxycarbonylmethyl) Nucleobases - Building Blocks for Peptide Nucleic Acids}

\author{
Guirong Qu *, Zhiguang Zhang, Haiming Guo, Mingwei Geng and Ran Xia \\ College of Chemistry and Environmental Science, Henan Normal University, Xinxiang 453007, P.R. \\ China; E-mails: zhiguang_zhang82@163.com; guohm518@hotmail.com; gengmingwei@126.com; \\ xiaran3324@163.com
}

* Author to whom correspondence should be addressed. E-mail: quguir@163.com

Received: 13 December 2006; in revised form: 11 February 2007 / Accepted: 17 February 2007 / Published: 19 March 2007

\begin{abstract}
A simple, rapid, and regioselective approach for the synthesis of N-(methoxycarbonylmethyl)- and N-(n-propoxycarbonylmethyl) nucleobases was developed. By using $\mathrm{DMF}$ as the solvent and in the presence of $\mathrm{K}_{2} \mathrm{CO}_{3}$ as the base, all the desired products were obtained in moderate yields within 8 min under microwave irradiation.
\end{abstract}

Keywords: Modified nucleoside analogues, microwave irradiation, peptide nucleic acids

\section{Introduction}

In recent years, modified nucleoside analogues have become of great interest due to their intriguing biological and pharmacological properties [1-5]. For example, a number of agents that exhibit potent anti-viral and anti-tumor activities such as AZT [6], Acyclovir [7-8], Neplanocin A [9], Peptide Nucleic Acids (PNA) [10-12] and so on have been prepared by modification of the carbohydrate ring of the natural nucleosides. PNA is a potent DNA mimic, in which the sugar-phosphate backbone of natural nucleic acids is replaced by a polyamide backbone. Since it was first reported by Nielsen and coworkers in 1991, PNA has attracted wide attention in medicinal chemistry for the development of gene therapy drugs or molecular probes. As a result, several groups have developed a variety of methods for the preparation of N-(alkoxycarbonylmethyl) nucleobases and their derivatives [13-17], 
which are important building blocks for PNA. However, the reported methods have some drawbacks, such as poor yields and regioselectivity, long reaction times and harsh reaction conditions.

In order to expand our research on the modification of nucleosides [18-20] and obtain these building blocks in higher yields with shorter reaction times and under milder reaction conditions, we turned our attention to microwave irradiation (MWI). The use of microwave-assisted organic syntheses has attracted considerable interest over the last two decades, leading to remarkable decreases in reaction times, significant enhancements of yields, easier workups and better regioselectivity [21-29]. Herein, we report a rapid, facile and practical protocol for the formation of $\mathrm{N}$ (methoxycarbonylmethyl)- and N-(n-propoxycarbonylmethyl) nucleobases.

\section{Results and Discussion}

Initially, we selected the reaction of uracil with methyl chloroacetate as a model system to investigate the influence of base and irradiation time on the yield, as summarized in Table 1.

Table 1. Optimization of Base and Irradiation Time ${ }^{\mathrm{a}}$.

\begin{tabular}{|c|c|c|c|}
\hline & & & \\
\hline Entry & Base & Irradiation time/min & Yield/\% ${ }^{b}$ \\
\hline 1 & DMAP & 6 & 52 \\
\hline 2 & $\mathrm{CH}_{3} \mathrm{ONa}$ & 6 & 41 \\
\hline 3 & $\mathrm{NaH}$ & 6 & 50 \\
\hline 4 & $\mathrm{~K}_{2} \mathrm{CO}_{3}$ & 6 & 64 \\
\hline 5 & $\mathrm{~K}_{2} \mathrm{CO}_{3}$ & 8 & 76 \\
\hline 6 & $\mathrm{~K}_{2} \mathrm{CO}_{3}$ & 9 & 74 \\
\hline 7 & $\mathrm{~K}_{2} \mathrm{CO}_{3}$ & 10 & 68 \\
\hline
\end{tabular}

All reactions were carried out in DMF, as it is an excellent solvent both for dissolving nucleobases and absorbing microwave energy. To our delight, 3a was obtained in 52\% yield by using DMAP as base (entry 1). Changing the base to $\mathrm{CH}_{3} \mathrm{ONa}$ or $\mathrm{NaH}$ only led to worse results (entries 2 and 3). An obvious yield improvement was observed when $\mathrm{K}_{2} \mathrm{CO}_{3}$ was employed (entry 4). Consequently, $\mathrm{K}_{2} \mathrm{CO}_{3}$ was selected as the best base, not only because it gave rise to the best results, but also because it is very cheap and easy to handle. The irradiation time had also significant effect on the yield, but it seemed that the reaction reached chemical equilibrium after being irradiated for some $8 \mathrm{~min}$, as only slight yield variations were detected after longer irradiation times (entries 5 and 6). With prolonged reaction times (entry 7) a lower yield of 3a resulted and some $\mathrm{N}^{3}$-alkylated product was obtained. Further screening of irradiation power and reaction temperatures confirmed that $250 \mathrm{~W}$ and $160{ }^{\circ} \mathrm{C}$ 
were the best conditions. With this promising procedure in hand, we then extended the scope of substrates to include other uracil derivatives, as outlined in Table 2.

Table 2. Alkylation of Various Uracil Derivatives in DMF under MWI ${ }^{\mathrm{a}}$.

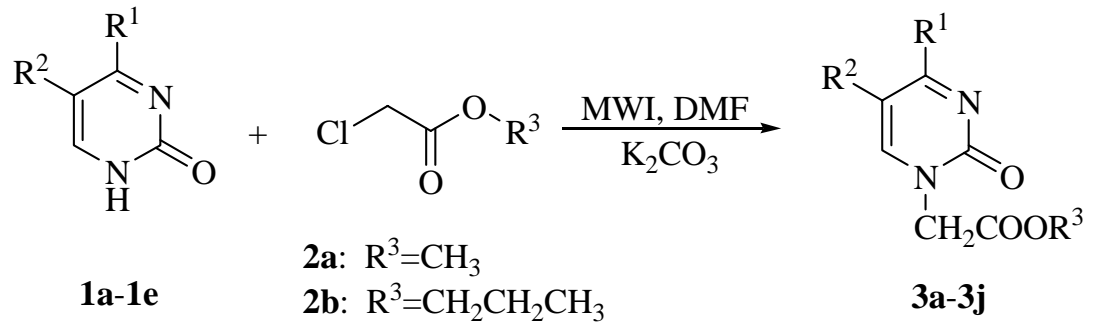

\begin{tabular}{ccccccc}
\hline Entry & Uracil derivative & $\boldsymbol{R}^{\mathbf{1}}$ & $\boldsymbol{R}^{\mathbf{2}}$ & $\boldsymbol{R}^{\mathbf{3}}$ & Product & Yield/\% \\
\hline 1 & $\mathbf{1 a}$ & $\mathrm{OH}$ & $\mathrm{H}$ & $\mathrm{CH}_{3}$ & 3a & 76 \\
2 & $\mathbf{1 b}$ & $\mathrm{OH}$ & $\mathrm{CH}$ & $\mathrm{CH}_{3}$ & $3 \mathbf{b}$ & 78 \\
3 & $\mathbf{1 c}$ & $\mathrm{OH}$ & $\mathrm{Cl}$ & $\mathrm{CH}_{3}$ & $3 \mathbf{c}$ & 73 \\
4 & $\mathbf{1 d}$ & $\mathrm{OH}$ & $\mathrm{I}$ & $\mathrm{CH}_{3}$ & $\mathbf{3 d}$ & 72 \\
5 & $\mathbf{1 e}$ & $\mathrm{NH}_{2}$ & $\mathrm{H}$ & $\mathrm{H}$ & $\mathbf{3 e}$ & $-{ }^{\mathrm{c}}$ \\
6 & $\mathbf{1 f}$ & $\mathrm{NHAc}$ & $\mathrm{H}$ & $\mathrm{CH}_{3}$ & $\mathbf{3 f}$ & 64 \\
7 & $\mathbf{1 a}$ & $\mathrm{OH}$ & $\mathrm{H}$ & $\mathrm{CH}_{2} \mathrm{CH}_{2} \mathrm{CH}_{3}$ & $\mathbf{3 g}$ & 70 \\
8 & $\mathbf{1 b}$ & $\mathrm{OH}$ & $\mathrm{CH}$ & $\mathrm{CH}_{2} \mathrm{CH}_{2} \mathrm{CH}_{3}$ & $\mathbf{3 h}$ & 67 \\
9 & $\mathbf{1 c}$ & $\mathrm{OH}$ & $\mathrm{Cl}$ & $\mathrm{CH}_{2} \mathrm{CH}_{2} \mathrm{CH}_{3}$ & $\mathbf{3 i}$ & 74 \\
10 & $\mathbf{1 d}$ & $\mathrm{OH}$ & $\mathrm{I}$ & $\mathrm{CH}_{2} \mathrm{CH}_{2} \mathrm{CH}_{3}$ & $\mathbf{3 j}$ & 68 \\
\hline
\end{tabular}

a Reaction conditions: 1 (2 mmol), 2 (6 mmol), base (2 mmol), DMF (5 mL), MWI $250 \mathrm{~W}$ (160 $\left.{ }^{\circ} \mathrm{C}\right)$;

${ }^{\mathrm{b}}$ Isolated yields based on $\mathbf{1}$.

${ }^{\mathrm{c}}$ the desired product $3 \mathbf{e}$ was not obtained.

To our delight, all the uracil derivatives were exclusively alkylated at $\mathrm{N}-1$, as confirmed by HMBC spectra, suggesting that our method was highly regioselective. It is worth mentioning that the same results were achieved when 2a was employed as the alkylating agent under the same conditions (entries 6-10). Substituting 5- $\mathrm{H}$ with $\mathrm{CH}_{3}, \mathrm{Cl}$, or I only resulted in slight variations in yield, indicating that no obvious substitutent-effect existed [26]. Disappointingly, alkylation of 1e gave very poor results and only starting material was recovered. In order to increase the solubility and prevent side reactions, $\mathrm{N}^{4}$-acetyl cytosine (1f) was then utilized as the precursor of $\mathbf{1 e}$ and treated with $\mathbf{2 a}$ as described above to afford $3 f$ in 64\% yield (entry 5).

Interestingly, the procedure developed for uracil derivatives also worked well for purine derivatives. As can be seen from Table 3, the length of alkyl chain in the chloroacetate reagent did not affect the yield. The target $\mathrm{N}^{9}$-alkylated products were produced in high regioselectivity and good yields. To our surprise, no obvious changes in yields were observed when the 6-Cl and 2- $\mathrm{H}$ in 4 a were substituted by 6-benzylamino (4c) and 2-Cl groups (4b), respectively. A possible explanation is that substitutents in these positions do not affect the electronic nature of N-9.

In order to investigate the capability and selectivity of our method compared with the conventional heating method, the formation of 3a was carried out in a pre-heated oil bath under the same conditions used with the microwave irradiation. It was shown the reaction afforded only 13\% yield after 8 min 
and $50 \%$ yield after $6 \mathrm{~h}$, and that the product was associated with the $\mathrm{N}^{3}$-alkylated byproduct, clearly indicating that our method was superior to the conventional method.

Table 3. Alkylation of Various Purine Derivatives in DMF under MWI ${ }^{\text {a }}$.

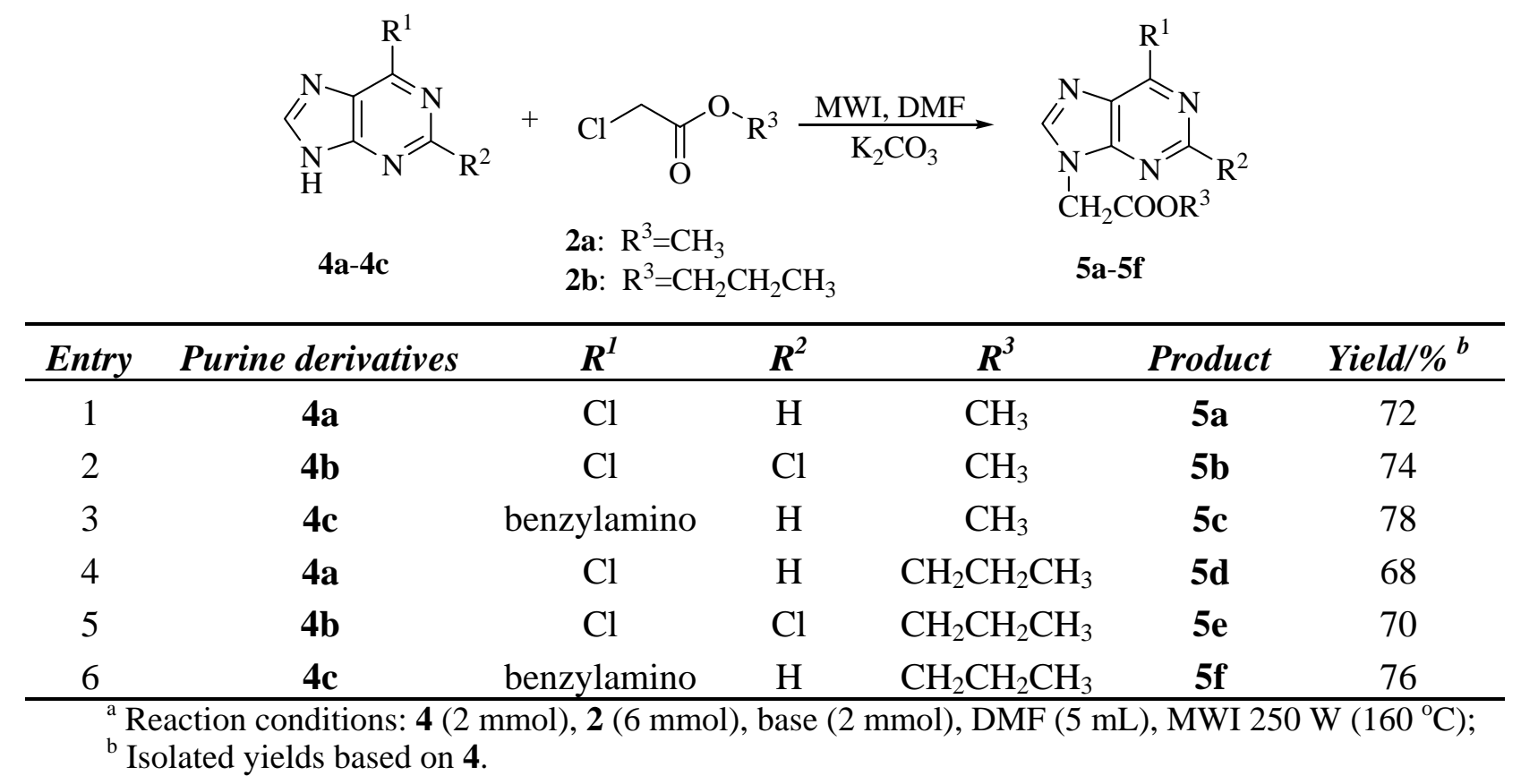

\section{Conclusions}

In summary, we have developed a procedure for the preparation of $\mathrm{N}$-(alkoxycarbonylmethyl) nucleobases that is rapid, simple and highly efficient in terms of yield and regioselectivity. Our method has several additional advantages, such as milder reaction conditions, short reaction times and lack of side products. The use of this method to synthesize other PNA building blocks is currently under study in our laboratory and the results will be reported in due course.

\section{Acknowledgements}

We are grateful for financial support from the Natural Science Foundation of China (No. 20372018) for financial support.

\section{Experimental Section}

\section{General}

All reagents and solvents were purchased from commercial sources and used without further purification. The nucleobases were a gift of Xinxiang Tuoxin Biochemical Technology \& Science Co. Ltd, P. R. China. Melting points were determined on an XRC-1 micro melting point apparatus and are uncorrected. ${ }^{1} \mathrm{H}$ - and ${ }^{13} \mathrm{C}-\mathrm{NMR}$ spectra were recorded in DMSO- $\mathrm{d}_{6}$ solutions on a Bruker DPX-400 spectrometer (at $400 \mathrm{MHz}$ and $100 \mathrm{MHz}$, respectively) using TMS as internal standard. High resolution mass spectra were obtained on the electrospray ionization (ESI) mass spectrometer. 
Elemental analyses were performed on an EA-1110 (CE Instruments) instrument. All reactions were performed in a commercially available single-mode microwave apparatus equipped with a high sensitivity IR sensor for temperature control and measurement (MAS-I, Sineo Microwave Chemical Technology Co. Ltd., Shanghai, China).

\section{General Procedure for the preparation of 3a (microwave method)}

A mixture of uracil (2 mmol, $0.224 \mathrm{~g}$ ), $\mathrm{K}_{2} \mathrm{CO}_{3}$ ( $2 \mathrm{mmol}, 0.276 \mathrm{~g}$ ) and methyl chloroacetate (6 mmol, $0.55 \mathrm{~mL}$ ) in DMF $(5 \mathrm{~mL})$ was placed in a $50 \mathrm{~mL}$ round-bottom glass flask. After being irradiated at $250 \mathrm{~W}\left(160{ }^{\circ} \mathrm{C}\right)$ for $8 \mathrm{~min}$, the reaction mixture was concentrated to dryness under reduced pressure and the residue was purified by column chromatography (1:1 ethyl acetatecyclohexane) to afford 1-(methoxycarbonylmethyl) uracil (3a) as a white power in 76\% yield; M.p.180-182 ${ }^{\circ} \mathrm{C}$ (ref. 30); ${ }^{1} \mathrm{H}-\mathrm{NMR} \delta: 3.69$ (s, 3H, $\mathrm{CH}_{3}$ ), 4.52 (s, 2H, $\mathrm{CH}_{2}$ ), 5.62 (d, 1H, J=8 Hz, H-5), 7.62 (d, 1H, J=8 Hz, H-6), 11.40 (s, 1H, H-3); ${ }^{13} \mathrm{C}-\mathrm{NMR} \delta: 48.7\left(\mathrm{CH}_{2}\right), 52.4\left(\mathrm{CH}_{3}\right), 101.3$ (C-5), 146.0 (C-6), 151.1 (C-2), 163.9 (C-4), $168.8(\mathrm{C}=\mathrm{O})$. The following compounds were similarly prepared:

1-(Methoxycarbonylmethyl) thymine (3b): Colorless needles; M.p. 189-190 ${ }^{\circ} \mathrm{C}$ (lit. [31] M.p. 189-191 ${ }^{0} \mathrm{C}$ ); ${ }^{1} \mathrm{H}-\mathrm{NMR} \delta: 1.76$ (d, $\left.J=0.4 \mathrm{~Hz}, \mathrm{CH}_{3}-5\right), 3.69$ (s, 3H, $\left.\mathrm{OCH}_{3}\right), 4.48$ (s, 2H, $\left.\mathrm{CH}_{2}\right), 7.50$ (d, $1 \mathrm{H}, J=0.4$ $\mathrm{Hz}, \mathrm{H}-6), 11.39$ (s, $1 \mathrm{H}, \mathrm{H}-3) ;{ }^{13} \mathrm{C}-\mathrm{NMR} \delta: 12.0\left(\mathrm{CH}_{3}-5\right), 48.5\left(\mathrm{CH}_{2}\right), 52.4\left(\mathrm{CH}_{3}\right), 108.8(\mathrm{C}-5), 141.7$ (C-6), 151.1 (C-2), 164.5 (C-4), 168.9 (C=O).

5-Chloro-1-(methoxycarbonylmethyl) uracil (3c): Colorless needles; M.p. 203-204 ${ }^{\circ} \mathrm{C} ;{ }^{1} \mathrm{H}-\mathrm{NMR} \delta$ : 3.68 (s, 3H, $\left.\mathrm{CH}_{3}\right), 4.53$ (s, 2H, $\mathrm{CH}_{2}$ ), 8.16 (s, $\left.1 \mathrm{H}, \mathrm{H}-6\right), 11.10$ (s, $\left.1 \mathrm{H}, \mathrm{H}-3\right) ;{ }^{13} \mathrm{C}-\mathrm{NMR} \delta 48.8\left(\mathrm{CH}_{2}\right)$, $52.6\left(\mathrm{CH}_{3}\right), 106.5$ (C-5), 143.2 (C-6), 150.2 (C-2), 159.6 (C-4), 168.5 (C=O); HR-MS calcd. for $\mathrm{C}_{7} \mathrm{H}_{7} \mathrm{ClN}_{2} \mathrm{O}_{4}$ 218.0094, found 218.0087; Anal. calcd. for $\mathrm{C}_{7} \mathrm{H}_{7} \mathrm{ClN}_{2} \mathrm{O}_{4}$ for C, 38.46, H, 3.23, N, 12.82, found $\mathrm{C}, 38.45, \mathrm{H}, 3.18, \mathrm{~N}, 12.72$.

5-Iodo-1-(methoxycarbonylmethyl) uracil (3d): Colorless plates; M.p. 197-199 ${ }^{\circ} \mathrm{C}[32]$; ${ }^{1} \mathrm{H}-\mathrm{NMR} \delta$ : 3.69 (s, 3H, $\left.\mathrm{CH}_{3}\right), 4.52$ (s, 2H, $\left.\mathrm{CH}_{2}\right), 8.20$ (s, $\left.1 \mathrm{H}, \mathrm{H}-6\right), 11.81$ (s, $\left.1 \mathrm{H}, \mathrm{H}-3\right) ;{ }^{13} \mathrm{C}-\mathrm{NMR} \delta: 48.6\left(\mathrm{CH}_{2}\right)$, $52.5\left(\mathrm{CH}_{3}\right), 68.4$ (C-5), 150.2 (C-6), 150.8 (C-2), 161.2 (C-4), $168.6(\mathrm{C}=\mathrm{O})$; HR-MS calcd. for $\mathrm{C}_{7} \mathrm{H}_{7} \mathrm{IN}_{2} \mathrm{O}_{4}$ 309.9450, found 309.9446; Anal. calcd. for $\mathrm{C}_{7} \mathrm{H}_{7} \mathrm{IN}_{2} \mathrm{O}_{4}$ for C, 27.12, $\mathrm{H}, 2.28, \mathrm{~N}, 9.04$, found C 27.16, H 2.23, N 9.08.

$N^{4}$-Acetyl-1-(methoxycarbonylmethyl) cytosine (3f): Colorless needles; M.p.186-188 ${ }^{\circ} \mathrm{C} ;{ }^{1} \mathrm{H}-\mathrm{NMR} \delta$ : 2.10 (s, 3H, $\mathrm{COCH}_{3}$ ), 3.68 (s, 3H, $\mathrm{OCH}_{3}$ ), 4.63 (s, 2H, $\mathrm{CH}_{2}$ ), 7.20 (d, 1H, J=7.6 Hz, H-5), 8.05 (d, 1H, $J=7.6 \mathrm{~Hz}, \mathrm{H}-5), 10.89$ (s, 1H, NH); ${ }^{13} \mathrm{C}-\mathrm{NMR} \delta: 24.5\left(\mathrm{COCH}_{3}\right), 50.8\left(\mathrm{OCH}_{3}\right), 52.4\left(\mathrm{NCH}_{2}\right), 95.4(\mathrm{C}-$ 5), 150.7 (C-6), 155.3 (C-2), 163.2 (C-4), $168.6\left(\mathrm{COCH}_{3}\right), 171.1(\mathrm{C}=\mathrm{O})$; HR-MS calcd. for $\mathrm{C}_{9} \mathrm{H}_{11} \mathrm{~N}_{3} \mathrm{O}_{4}$ 225.0750, found 225.0746; Anal. calcd. for $\mathrm{C}_{9} \mathrm{H}_{11} \mathrm{~N}_{3} \mathrm{O}_{4}$ for C, 48.00; H, 4.92; N, 18.66; O, 28.42, found C, 47.91, H, 4.87, N 18.59 .

1-(n-Propoxycarbonylmethyl) uracil (3g): Colorless plates; M.p. 124-125 ${ }^{\circ} \mathrm{C} ;{ }^{1} \mathrm{H}-\mathrm{NMR} \delta$ : 0.88 (t, $3 \mathrm{H}$, $J=7.2 \mathrm{~Hz}, \mathrm{CH}_{3}$ ), 1.60 (m, 2H, $\mathrm{CH}_{2} \mathrm{CH}_{3}$ ), 4.07 (t, 2H, J=7.2 Hz, $\mathrm{OCH}_{2}$ ), 4.52 (s, 2H, $\mathrm{NCH}_{2}$ ), 5.61 (d, 1H, $J=8 \mathrm{~Hz}, \mathrm{H}-5$ ), 7.62 (d, 1H, J=8 Hz, H-6), 11.38 (s, 1H, H-3); ${ }^{13} \mathrm{C}-\mathrm{NMR} \delta: 10.3\left(\mathrm{CH}_{3}\right), 21.6$ 
$\left(\mathrm{CH}_{2} \mathrm{CH}_{3}\right), 48.8\left(\mathrm{NCH}_{2}\right), 66.7\left(\mathrm{OCH}_{2}\right), 101.2$ (C-5), 146.0 (C-6), 151.1 (C-2), 163.9 (C-4), 168.3 (C=O); HR-MS calcd. for $\mathrm{C}_{9} \mathrm{H}_{12} \mathrm{~N}_{2} \mathrm{O}_{4}$ 212.0797, found 212.0290; Anal. calcd. for $\mathrm{C}_{9} \mathrm{H}_{12} \mathrm{~N}_{2} \mathrm{O}_{4}$ for C, 50.94; H, 5.70; N, 13.20, found C, 50.90; H, 5.61; N, 13.30 .

1-(n-Propoxycarbonylmethyl) thymine (3h) Colorless plates; M.p. $140-141{ }^{\circ} \mathrm{C} ;{ }^{1} \mathrm{H}-\mathrm{NMR} \delta: 0.88$ (t, $3 \mathrm{H}$, $\left.J=7.2 \mathrm{~Hz}, \mathrm{CH}_{2} \mathrm{CH}_{3}\right), 1.60$ (m, 2H, $\mathrm{CH}_{2} \mathrm{CH}_{3}$ ), 1.76 (s, 3H, $\left.\mathrm{CH}_{3}-5\right), 4.06$ (t, 2H, J=7.2 Hz, $\mathrm{OCH}_{2}$ ), 4.47 (s, 2H, $\mathrm{NCH}_{2}$ ), 7.50 (d, $\left.1 \mathrm{H}, J=1.2 \mathrm{~Hz}, \mathrm{H}-6\right), 11.36$ (s, $\left.1 \mathrm{H}, \mathrm{H}-3\right) ;{ }^{13} \mathrm{C}-\mathrm{NMR} \delta: 10.3\left(\mathrm{CH}_{2} \mathrm{CH}_{3}\right), 12.0$ $\left(\mathrm{CH}_{3}-5\right), 21.6\left(\mathrm{CH}_{2} \mathrm{CH}_{3}\right), 48.6\left(\mathrm{NCH}_{2}\right), 66.6\left(\mathrm{OCH}_{2}\right), 108.7$ (C-5), 141.7 (C-6), 151.1 (C-2), $164.5(\mathrm{C}-$ 4), $168.4(\mathrm{C}=\mathrm{O})$; HR-MS calcd. for $\mathrm{C}_{10} \mathrm{H}_{14} \mathrm{~N}_{2} \mathrm{O}_{4}$ 226.0954, found 226.0950; Anal. calcd. for $\mathrm{C}_{10} \mathrm{H}_{14} \mathrm{~N}_{2} \mathrm{O}_{4}$ for C, 53.09; H, 6.24; N, 12.38, found C, 53.00; H, 6.15; N, 12.30 .

5-Chloro-1-(n-propoxycarbonylmethyl) uracil (3i) White power; M.p. 148-149 ${ }^{\circ} \mathrm{C} ;{ }^{1} \mathrm{H}-\mathrm{NMR} \delta$ : 0.88 (t, $3 \mathrm{H}, J=7.2 \mathrm{~Hz}, \mathrm{CH}_{2} \mathrm{CH}_{3}$ ), 1.60 (m, 2H, $\left.\mathrm{CH}_{2} \mathrm{CH}_{3}\right), 4.06$ (t, 2H, J=7.2 Hz, OCH$)_{2}, 4.52$ (s, 2H, $\mathrm{NCH}_{2}$ ), 8.16 (d, $1 \mathrm{H}, J=4 \mathrm{~Hz}, \mathrm{H}-6), 11.97$ (s, $1 \mathrm{H}, \mathrm{H}-3) ;{ }^{13} \mathrm{C}-\mathrm{NMR} \delta: 10.3\left(\mathrm{CH}_{2} \mathrm{CH}_{3}\right), 21.6\left(\mathrm{CH}_{2} \mathrm{CH}_{3}\right), 48.9$ $\left(\mathrm{NCH}_{2}\right), 66.8\left(\mathrm{OCH}_{2}\right), 106.5$ (C-5), 143.3 (C-6), 150.3 (C-2), 159.6 (C-4), 168.0 (C=O); HR-MS calcd. for $\mathrm{C}_{9} \mathrm{H}_{11} \mathrm{ClN}_{2} \mathrm{O}_{4}$ 246.0407, found 246.0401; Anal. calcd. for $\mathrm{C}_{9} \mathrm{H}_{11} \mathrm{ClN}_{2} \mathrm{O}_{4}$ for C, 43.83; H, 4.50; N, 11.36, found C, 43.78; H, 4.41; N, 11.30 .

5-Iodo-1-(n-propoxycarbonylmethyl) uracil (3j) Colorless needles; M.p. 156-157 ${ }^{\circ} \mathrm{C} ;{ }^{1} \mathrm{H}-\mathrm{NMR} \delta$ : 0.88 (t, 3H, J=7.2 Hz, $\mathrm{CH}_{3}$ ), 1.60 (m, 2H, $\left.\mathrm{CH}_{2} \mathrm{CH}_{3}\right), 4.07$ (t, 2H, J=7.2 Hz, $\mathrm{OCH}_{2}$ ), 4.52 (s, 2H, $\mathrm{NCH}_{2}$ ), 8.22 (d, $1 \mathrm{H}, \mathrm{J}=4 \mathrm{~Hz}, \mathrm{H}-6), 11.80$ (s, $1 \mathrm{H}, \mathrm{H}-3) ;{ }^{13} \mathrm{C}-\mathrm{NMR} \delta: 10.3\left(\mathrm{CH}_{2} \mathrm{CH}_{3}\right), 21.7\left(\mathrm{CH}_{2} \mathrm{CH}_{3}\right), 48.7$ $\left(\mathrm{NCH}_{2}\right), 66.8\left(\mathrm{OCH}_{2}\right), 68.3$ (C-5), 150.2 (C-6), 150.8 (C-2), 161.2 (C-4), 168.1 (C=O); HR-MS calcd. for $\mathrm{C}_{9} \mathrm{H}_{11} \mathrm{IN}_{2} \mathrm{O}_{4} 337.9763$, found 337.9759; Anal. calcd. for $\mathrm{C}_{9} \mathrm{H}_{11} \mathrm{IN}_{2} \mathrm{O}_{4}$ for C, 31.97; H, 3.28; N 8.29, found C, 31.89; H, 3.20; N, 8.25.

6-Chloro-9-(methoxycarbonylmethyl) purine (5a) Colorless plates; M.p. $109-110{ }^{\circ} \mathrm{C}$ [33]; ${ }^{1} \mathrm{H}-\mathrm{NMR} \delta$ : 3.73 (s, 3H, $\mathrm{CH}_{3}$ ), 5.29 (s, 2H, $\mathrm{CH}_{2}$ ), 8.68 (s, $\left.1 \mathrm{H}, \mathrm{H}-8\right), 8.80$ (s, $\left.1 \mathrm{H}, \mathrm{H}-2\right) ;{ }^{13} \mathrm{C}-\mathrm{NMR} \delta: 44.74\left(\mathrm{CH}_{2}\right)$, $52.8\left(\mathrm{CH}_{3}\right), 130.7$ (C-5), 148.0 (C-8), 149.4 (C-4), 152.0 (C-6), 152.2 (C-2), $168.0(\mathrm{C}=\mathrm{O})$.

2,6-Dichloro-9-(methoxycarbonylmethyl) purine (5b) Colorless plates; M.p. 142-144 ${ }^{\circ} \mathrm{C}[34]$; ${ }^{1} \mathrm{H}-$ NMR $\delta: 3.74$ (s, 3H, $\left.\mathrm{CH}_{3}\right), 5.27$ (s, 2H, $\left.\mathrm{CH}_{2}\right), 8.71$ (s, $\left.1 \mathrm{H}, \mathrm{H}-8\right) ;{ }^{13} \mathrm{C}-\mathrm{NMR} \delta: 44.48\left(\mathrm{CH}_{2}\right), 52.9\left(\mathrm{CH}_{3}\right)$, 130.3 (C-5), 149.0 (C-8), 150.1 (C-4), 151.5 (C-6), 153.7 (C-2), 167.8 (C=O).

6-Benzylamino-9-(methoxycarbonylmethyl) purine (5c) Pale yellow power; M.p. $138-139{ }^{\circ} \mathrm{C} ;{ }^{1} \mathrm{H}-\mathrm{NMR}$ $\delta$ : 3.70 (s, 3H, $\mathrm{CH}_{3}$ ), 4.72 (s, 2H, $\mathrm{CH}_{2} \mathrm{Ph}$ ), 5.10 (s, 2H, NCH ), 7.19 7.36 (m, 5H, Ph), 8.14 (s, 1H, H8), 8.19 (s, 1H, H-2), 8.39 (s, 1H, NH); ${ }^{13} \mathrm{C}-\mathrm{NMR} \delta: 43.1\left(\mathrm{CH}_{2} \mathrm{Ph}\right), 44.0\left(\mathrm{NCH}_{2}\right), 52.6\left(\mathrm{CH}_{3}\right), 119.5$ (C-5), 126.8, 127.7, 128.4 (Ph), 140.3 (C-8), 141.4 (C-4), 152.8 (C-2), 154.6 (C-6), 168.6 (C=O); HRMS calcd. for $\mathrm{C}_{15} \mathrm{H}_{15} \mathrm{~N}_{5} \mathrm{O}_{2}$ 297.1226, found 297.1220; Anal. calcd. for $\mathrm{C}_{15} \mathrm{H}_{15} \mathrm{~N}_{5} \mathrm{O}_{2}$ for $\mathrm{C}$, 60.60; $\mathrm{H}$, 5.09; N, 23.56, found C, 60.53; H, 5.02; N, 23.60.

6-Chloro-9-(n-propoxycarbonylmethyl) purine (5d) Yellow power; M.p. 56-58 ${ }^{\circ} \mathrm{C} ;{ }^{1} \mathrm{H}-\mathrm{NMR} \delta: 0.84$ (t, $\left.3 \mathrm{H}, J=7.2 \mathrm{~Hz}, \mathrm{CH}_{3}\right), 1.59$ (m, 2H, $\left.\mathrm{CH}_{2} \mathrm{CH}_{3}\right), 4.09$ (t, 2H, J=7.2 Hz, OCH $), 5.29$ (s, 2H, $\left.\mathrm{NCH}_{2}\right), 8.69$ (s, $1 \mathrm{H}, \mathrm{H}-8), 8.19$ (s, 1H, H-2); ${ }^{13} \mathrm{C}-\mathrm{NMR} \delta: 10.2\left(\mathrm{CH}_{2} \mathrm{CH}_{3}\right), 21.5\left(\mathrm{CH}_{2} \mathrm{CH}_{3}\right), 44.8\left(\mathrm{NCH}_{2}\right), 67.1$ 
( $\mathrm{OCH}_{2}$ ), 130.7 (C-5), 148.0 (C-8), 149.4 (C-4), 152.0 (C-6), 152.2 (C-2), 167.5 (C=O); HR-MS calcd. for $\mathrm{C}_{10} \mathrm{H}_{11} \mathrm{ClN}_{4} \mathrm{O}_{2}$ 254.0571, found 254.0562; Anal. calcd. for $\mathrm{C}_{10} \mathrm{H}_{11} \mathrm{ClN}_{4} \mathrm{O}_{2}$ for $\mathrm{C}$, 47.16; $\mathrm{H}, 4.35$; , 22.00, found C, 47.09; H, 4.27; N, 21.89.

2,6-Dichloro-9-(n-propoxycarbonylmethyl) purine (5e) Colorless rod-like crystals; M.p. 117-119 ${ }^{\circ} \mathrm{C}$; ${ }^{1} \mathrm{H}-\mathrm{NMR} \delta: 0.86\left(\mathrm{t}, 3 \mathrm{H}, J=7.2 \mathrm{~Hz}, \mathrm{CH}_{2} \mathrm{CH}_{3}\right), 1.60\left(\mathrm{~m}, 2 \mathrm{H}, \mathrm{CH}_{2} \mathrm{CH}_{3}\right), 4.11\left(\mathrm{t}, 2 \mathrm{H}, J=7.2 \mathrm{~Hz}, \mathrm{OCH}_{2}\right)$, 5.27 (s, 2H, NCH$), 8.71$ (s, $1 \mathrm{H}, \mathrm{H}-8) ;{ }^{13} \mathrm{C}-\mathrm{NMR} \delta: 10.2\left(\mathrm{CH}_{3}\right), 21.6\left(\mathrm{CH}_{2} \mathrm{CH}_{3}\right), 45.0\left(\mathrm{NCH}_{2}\right), 67.2$ $\left(\mathrm{OCH}_{2}\right), 130.3$ (C-5), 149.0 (C-8), 150.1 (C-4), 151.5 (C-6), 153.8 (C-2), 167.3 (C=O); HR-MS calcd. for $\mathrm{C}_{10} \mathrm{H}_{10} \mathrm{Cl}_{2} \mathrm{~N}_{4} \mathrm{O}_{2}$ 288.0181, found 288.0178; Anal. calcd. for $\mathrm{C}_{10} \mathrm{H}_{10} \mathrm{Cl}_{2} \mathrm{~N}_{4} \mathrm{O}_{2}$ for $\mathrm{C}$, 41.54; $\mathrm{H}$, 3.49; N, 19.38, found C, 41.45; H, 3.41; N, 19.46 .

6-Benzylamino-9-(n-propoxycarbonylmethyl) purine (5f) Colorless plates; M.p. 179-180 ${ }^{\circ} \mathrm{C} ;{ }^{1} \mathrm{H}-\mathrm{NMR}$ $\delta: 0.85$ (t, 3H, J=7.2 Hz, $\mathrm{CH}_{3}$ ), $1.59\left(\mathrm{~m}, 2 \mathrm{H}, \mathrm{CH}_{2} \mathrm{CH}_{3}\right), 4.07$ (t, 2H, J=7.2 Hz, $\mathrm{OCH}_{2}$ ), 4.72 (s, 2H, $\mathrm{CH}_{2} \mathrm{Ph}$ ), 5.09 (s, 2H, NCH ), 7.19 7.36 (m, 5H, Ph), 8.14 (s, 1H, H-8), 8.19 (s, 1H, H-2), 8.34 (s, 1H, $\mathrm{NH}) ;{ }^{13} \mathrm{C}-\mathrm{NMR} \delta$ : $10.2\left(\mathrm{CH}_{3}\right), 21.6\left(\mathrm{CH}_{2} \mathrm{CH}_{3}\right), 43.1\left(\mathrm{CH}_{2} \mathrm{Ph}\right), 44.1\left(\mathrm{NCH}_{2}\right), 66.8\left(\mathrm{OCH}_{2}\right), 118.8(\mathrm{C}-5)$, 126.8, 127.3, 128.3 (Ph), 140.3 (C-8), 141.4 (C-4), 152.8 (C-6), 154.8 (C-2), 168.1 (C=O); HR-MS calcd. for $\mathrm{C}_{17} \mathrm{H}_{19} \mathrm{~N}_{5} \mathrm{O}_{2} 325.1539$, found 325.1536; Anal. calcd. for $\mathrm{C}_{17} \mathrm{H}_{19} \mathrm{~N}_{5} \mathrm{O}_{2}$ for C, 62.75; H, 5.89; N, 21.52, found C, 62.68; H, 5.80; N, 21.60.

Synthesis of 3a (conventional method)

A mixture of uracil (2 mmol, $0.224 \mathrm{~g}$ ), $\mathrm{K}_{2} \mathrm{CO}_{3}(2 \mathrm{mmol}, 0.276 \mathrm{~g}$ ) and methyl chloroacetate (6 mmol, $0.55 \mathrm{~mL}$ ) in DMF (5 mL), contained in a $50 \mathrm{~mL}$ round-bottom glass flask, was stirred under reflux in an oil-bath $\left(160^{\circ} \mathrm{C}\right)$ for 8 min or 6 h. The workups were performed as described above for the microwave method.

\section{References}

1. Haines, D. R.; Tseng, C. K. H.; Marquez, V. E. Synthesis and biological activity of unsaturated carboacyclic purine nucleoside analogs. J. Med. Chem. 1987, 30, 943-947.

2. Bisacchi, G. S.; Singh, J.; Godfrey, J. D. Jr.; Kissick, T. P.; Mitt, T.; Malley, M. F.; Di Marco, J. D.; Gougoutas, J. Z.; Mueller, R. H.; Zahler, R. Regioselective Coupling of Tetraalkylammonium Salts of 6-Iodo-2-aminopurine to a Cyclobutyl Triflate: Efficient Preparation of Homochiral BMS-180,194, a Potent Antiviral Carbocyclic Nucleoside. J. Org. Chem. 1995, 60, 2902-2905.

3. Paoli, M.L.; Piccini, S.; Rodriquez, M.; Sega, A. Sensible Improvements Induced by Ionic Liquids in the Reaction of Modified Carbasugars with Bases for the Building of Constrained Carbanucleosides. J. Org. Chem. 2004, 69, 2881-2883.

4. Freer, R.; Geen, G. R.; Ramsay, T. W.; Share, A. C.; Slater, G. R.; Smith, N. M. A New Route to Famciclovir via Palladium Catalysed Allylation. Tetrahedron 2000, 56, 4589-4595.

5. Lanver, A.; Schmalz, H.-G. Microwave-Assisted Amination of a Chloropurine Derivative in the Synthesis of Acyclic Nucleoside Analogues. Molecules 2005, 10, 508-515.

6. Mitsuya, H.; Weinhold, K.J.; Furman, P.A. 3'-Azido-3'-deoxythymidine (BW A509U): An antiviral agent that inhibits the infectivity and cytopathic effect of human T-lymphotropic virus 
type III/lymphadenopathy-associated virus in vitro. Pro. Natl. Acad. Sci. USA. 1985, 82, 70967100.

7. Vandenriessche, F.; Snoeck, R.; Janssen, G.; Hoogmartens, J.; Aerschot, A. V.; De Clercq, E.; Herdewijn, P. Synthesis and antiviral activity of acyclic nucleosides with a 3(S),5dihydroxypentyl or 4(R)-methoxy-3 (S),5-dihydroxypentyl side chain. J. Med. Chem. 1992, 35, 1458-1465.

8. Schaeffer, H.J.; Beauchamp, L.; De Miranda, P. 9-(2-Hydroxyethoxymethyl)guanine activity against viruses of the herpes group. Nature 1978, 272, 583-585.

9. Ono, M.; Nishimura, K.; Tsubouchi, H.; Nagaoka, Y.; Tomioka, K. Total Synthesis of (-)Neplanocin A by Using Lithium Thiolate-Initiated Michael-Aldol Tandem Cyclization Reaction. J. Org. Chem. 2001, 66, 8199-8203.

10. Nielsen, P.E.; Egholm, M.; Berg, R.H.; Buchardt, O. Sequence-selective recognition of DNA by strand displacement with thymine-substituted polyamide. Science 1991, 254, 1497-1500.

11. Egholm, M.; Buchardt, O.; Christensen, L.; Behrens, C.; Freier, S.M.; Driver, D.A.; Berg, R.H.; Kim, S.K.; Norden, B.; Nielsen, P.E. PNA hybridizes to complementary oligonucleotides obeying the Watson-Crick hydrogen-bonding rules. Nature 1993, 365, 566-568.

12. Nielsen, P. E. Peptide Nucleic Acid. A Molecule with Two Identities. Acc. Chem. Res. 1999, 32, 624-630.

13. Austin, R. E.; Waldraff, C.; Al-Obeidi, F. Microwave assisted solid-phase synthesis of trisubstituted 2-(2,6-purin-9-yl) acetamides. Tetrahedron Lett. 2005, 46, 2873-2875.

14. Dueholm, K.L.; Egholm, M.; Behrens, C.; Christensen, L.; Hansen, H.F.; Vulpius, T.; Petersen, K.H.; Berg, R.H.; Nielsen, P.E.; Buchardt, O. Synthesis of Peptide Nucleic Acid Monomers Containing the Four Natural Nucleobases: Thymine, Cytosine, Adenine, and Guanine and Their Oligomerization. J. Org. Chem. 1994, 59, 5767-5773.

15. Thomson, S.A.; Josey, J.A.; Cadilla, R.; Gaul, M.D.; Hassman, C.F.; Luzzio, M.J.; Pipe, A.J.; Reed, K.L.; Ricca, D.J.; Wiethe, R.W.; Noble, S.A. Fmoc mediated synthesis of peptide nucleic acids. Tetrahedron 1995, 51, 6179-6194.

16. Egholm, M.; Buchardt, O.; Nielsen, P.E.; Berg, R.H. Peptide nucleic acids (PNA). Oligonucleotide analogs with an achiral peptide backbone. J. Am. Chem. Soc. 1992, 114, 18951897.

17. Will, D.W.; Breipohl, G.; Langner, D.; Knolle, J.; Uhlmann, E. The synthesis of polyamide nucleic acids using a novel monomethoxytrityl protecting-group strategy. Tetrahedron 1995, 51, 12069-12082.

18. Qu, G.-R.; Li, Y.; Han, S.-H. Microwave assisted synthesis of N-(ethoxycarbonylmethyl) nucleobases: Building blocks for PNAs. J. Chem. Res. 2005, 167-168.

19. Qu, G.-R.; Han, S.-H; Zhang, Z.; Geng, M.; Xue, F. Microwave assisted synthesis of 6-substituted aminopurine analogs in water. J. Braz. Chem. Soc. 2006, 17, 915-922.

20. Qu, G.-R.; Han, S.-H; Zhang, Z.; Geng, M.; Xue, F. Microwave-assisted regioselective synthesis of acyclic nucleosides through an alkylating reaction with 2-oxa-1,4-butanediol diacetate. Can. J. Chem. 2006, 84, 819-824.

21. Kappe, C.O. Controlled microwave heating in modern organic synthesis. Angew. Chem., Int. Ed. 2004, 43, 6250-6284. 
22. Loupy, A. Microwaves in organic synthesis; Wiley-VCH: Weinheim, 2002; pp. 253-290.

23. Ricardo, A.W.; Neves, F.; Rajendra, M.S. A Handy and Solventless Direct Route to Primary 3-[3Aryl)-1,2,4-oxadiazol-5-yl]propionamides Using Microwave Irradiation. Molecules 2006, 11, 318-324.

24. Varma, R.S. Solvent-free organic syntheses: Using supported reagents and microwave irradiation. Green Chem. 1999, 1, 43-55.

25. Liu, G.; Yang, S.; Song, B.; Xue, W.; Hu, D.; Jin, L.; Lu, P. Microwave Assisted Synthesis of NArylheterocyclic Substituted-4-aminoquinazoline Derivatives. Molecules 2006, 11, 272-278.

26. Khalafi-Nezhad, A.; Zare, A.; Parhami, A.; Soltani Rad, M.N.; Nejabat, G.R. Microwave-assisted N-nitroarylation of Some Pyrimidine and Purine Nucleosides. Can. J. Chem. 2006, 84, 979-985.

27. Le, H.P.; Muller, C.E. Rapid Microwave-assisted Fluorination Yielding novel 5'-deoxy-5'Fluorouridine Derivatives. Bioorg. Med. Chem. Lett. 2006, 16, 6139-6142.

28. Paolini, L.; Petricci, E.; Corelli, F.; Botta, M. Microwave-Assisted C-5 Iodination of Substituted Pyrimidinones and Pyrimidine Nucleosides. Synthesis 2003, 1039-1042.

29. Khalafi-Nezhad, A.; Zarea, A.; Soltani Rad, M.N.; Mokhtari, B.; Parhami, A. MicrowaveAssisted Michael Addition of Some Pyrimidine and Purine Nucleobases with $\alpha, \beta$-Unsaturated Esters: A Rapid Entry into Carboacyclic Nucleoside Synthesis. Synthesis 2005, 419-424.

30. Wyrzykiewicz, E.; Kazimierczuk, Z. An electron-impact mass spectral (EIMS) study of N-1 and C-6 carboxyalkyl- and alkoxycarbonylalkyl-substituted derivatives of uracil. J. Heterocycl. Chem. 1998, 35, 349-358.

31. Aldrian-Herrada, G.; Rabie, A.; Wintersteiger, R.; Brugidou, J. Solid-phase Synthesis of Peptide Nucleic Acid (PNA) Monomers and Their Oligomerization Using Disulphide Anchoring Linkers. J. Peptide Sci. 1998, 4, 266-281.

32. Bergmann, F.; Herrmann, R.; Seidel, C.; Koch, T. Novel monomer elements for marking peptidic nucleic acids. WO Patent 9842735 (1998).

33. Kim, B. Y.; Ahn, J. B.; Lee, H. W.; Kang, S. K.; Lee, J. H.; Shin, J. S.; Ahn, S. K.; Hong, C. I.; Yoon, S. S. Synthesis and biological activity of novel substituted pyridines and purines containing 2,4-thiazolidinedione. Eur. J. Med. Chem. 2004, 39, 433-447.

34. Brik, A.; Wu, C-Y; Best, M. D.; Wong, C-H. Tetrabutylammonium fluoride-assisted rapid N9alkylation on purine ring: Application to combinatorial reactions in microtiter plates for the discovery of potent sulfotransferase inhibitors in situ. Bioorg. \& Med. Chem. 2005, 13, 4622-4626.

Sample Availability: Samples of the compounds mentioned are available from authors.

(C) 2007 by MDPI (http://www.mdpi.org). Reproduction is permitted for noncommercial purposes. 